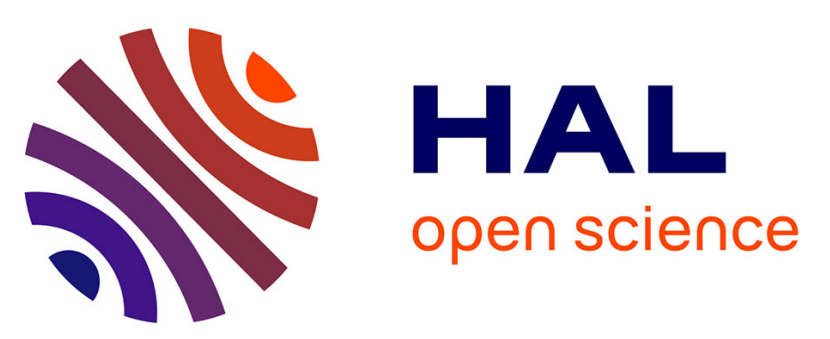

\title{
Local controllability and attitude stabilization of multirotor UAVs: Validation on a coaxial octorotor
}

Majd Saied, Hassan Shraim, Benjamin Lussier, Isabelle Fantoni, Clovis Francis

\section{To cite this version:}

Majd Saied, Hassan Shraim, Benjamin Lussier, Isabelle Fantoni, Clovis Francis. Local controllability and attitude stabilization of multirotor UAVs: Validation on a coaxial octorotor. Robotics and Autonomous Systems, 2017, 91, pp.128-138. 10.1016/j.robot.2017.01.007 . hal-01462104

\author{
HAL Id: hal-01462104 \\ https://hal.science/hal-01462104
}

Submitted on 8 Feb 2017

HAL is a multi-disciplinary open access archive for the deposit and dissemination of scientific research documents, whether they are published or not. The documents may come from teaching and research institutions in France or abroad, or from public or private research centers.
L'archive ouverte pluridisciplinaire HAL, est destinée au dépôt et à la diffusion de documents scientifiques de niveau recherche, publiés ou non, émanant des établissements d'enseignement et de recherche français ou étrangers, des laboratoires publics ou privés. 


\title{
Local Controllability and Attitude Stabilization of multirotor UAVs: Validation on a coaxial Octorotor
}

\author{
Majd Saied ${ }^{\mathrm{a}, \mathrm{b}}$, Hassan Shraim ${ }^{\mathrm{b}}$, Benjamin Lussier ${ }^{\mathrm{a}}$, Isabelle Fantoni ${ }^{\mathrm{a}}$, \\ Clovis Francis ${ }^{\mathrm{b}}$ \\ ${ }^{a}$ Sorbonne Universités, Université de Technologie de Compiègne, CNRS, UMR 7253 \\ Heudiasyc, 60200 Compiègne, France, Email: \{majd.saied, benjamin.lussier, \\ isabelle.fantoni\}@hds.utc.fr \\ ${ }^{b}$ Université Libanaise, Faculté de Génie, Centre de Recherche Scientifique en Ingénierie \\ (CRSI), Liban, Email: \{cfrancis, hassan.shraim\}@ul.edu.lb
}

\begin{abstract}
This paper addresses the attitude controllability problem for a multirotor unmanned aerial vehicle (UAV) in case of one or several actuators failures. The small time local controllability (STLC) of the system attitude dynamics is analysed using the nonlinear controllability theory with unilateral control inputs. This analysis considers different actuators configurations and compares their fault tolerance capabilities regarding actuators failures. Analytical results are then validated experimentally on a coaxial octorotor. A stabilization control law is applied on the coaxial configuration under one, two, three and four motors failures, when the system is controllable. Real-time experimental results demonstrate the effectiveness of the applied strategy.

Keywords: Fault Tolerant Control, Nonlinear Controllability, Unmanned aerial vehicles, Octorotor.
\end{abstract}

\section{Introduction}

Nowadays, a significant interest appears in the attitude control problem of multirotor unmanned aerial vehicles (UAV) under actuators failures. A desirable outcome consists in keeping a controllable attitude after one or more actuators failures, preventing the UAV from flipping over and crashing. For quadrotors, the attitude control problem after partial propellers failures has been investigated in several works and a wide class of Fault Tolerant Control methods has been proposed to stabilize the vehicle (Zhang et al. (2013), 
Chamseddine et al. (2012), Ranjbaran \& Khorasani (2010), etc.). However, a complete propeller's failure in a quadrotor results unavoidably in the loss of the system full controllability. The authors in (Mueller \& D'Andrea (2014)) demonstrated and validated the controllability of the reduced attitude in case of one, two and three failures where the control of the yaw state is neglected. Multirotors with redundant actuators have been proposed as a solution to this controllability loss.

Different octorotors configurations exist, among them we list the coaxial counter-rotating (Saied et al. (2015)), the PNPNPNPN (P:positive, N:negative) star-shaped or the PPNNPPNN star-shaped (Alwi \& Edwards (2013)) configurations, each with different fault tolerant capabilities. The investigation of dynamics stabilization of these different configurations begin with the theoretical establishment of the necessary and sufficient conditions for the system controllability and the control reconfigurability. A multirotor is a nonlinear system having positive control inputs since the rotors only provide a unidirectional thrust (we consider only in this study fixed-pitch rotors contributing to the total vertical produced thrust). Thus classical controllability theory of linear systems will have a major limitation if applied to a multirotor: the unilateral constraints do not pass the Kalman Rank Test (Sontag (1991)). This limitation is particularly problematic when dealing with multiple failures since we are working on an over-actuated system. In this paper, we propose the use of the non linear control theory with unilateral control inputs to assess the controllability of different configurations of octorotors. Based on this study, an experimental work is used to confirm the controllability of a coaxial counter-rotating octorotor under some actuators failures, using a control mixing and a feedback controller based on saturation functions.

\section{Methodology}

The controllability problem for linear systems has been actively developed in the literature. The existing methodologies are based on the Kalman rank criteria (Sontag (1991)). However, studying the controllability of a nonlinear system is more complex. A nonlinear system is said to be controllable if there exist admissible control inputs that will bring the system between two arbitrary states in a finite time. A general set of necessary and sufficient conditions for controllability of these systems does not currently exist. Instead, the controllability is studied by investigating the local behaviour of the system near equilibrium points. The simplest approach is to linearise 
the system around an equilibrium point and then to apply the Kalman Rank Test. However, this is not a necessary proof since a nonlinear system can be controllable even if though its linearisation is not.

A necessary condition for controllability from an initial state is given by computing the accessibility algebra $\Delta$ constructed by Lie Brackets. However, this is not a sufficient condition since it only infers conclusions on the dimension of the reachable space from the initial one. Small Time Local Controllability is a stronger property than controllability. It means that the system can be steered in any direction in a small amount of time. For second-order systems, STLC is only possible from equilibrium states (Sussmann (1987)). Sussmann presented sufficient conditions for STLC in (Sussmann (1987)) and they will be summarized later.

However, having constraints on the inputs renders these conditions invalid for testing the controllability and other tools are then necessary for this purpose. Goodwine proposed in (Goodwine \& Burdick (1996)) a method for the controllability of systems with unilateral control inputs. We use these results in our paper to investigate the possibility of stabilizing the octorotor after multiple successive failures. Few works studied the controllability of multirotors. In (Schneider et al. (2012)), the Attainable Control Set method is used for the study of static controllability. It is based on the definition of the limits in thrust and torque that can be allocated while satisfying the speed constraints of the motors. In (Du et al. (2014)), the authors proposed the use of a simplified test based on Brammer works (Brammer (1972)) to prove the controllability of linear systems with positive inputs. For this purpose, the linear dynamical model of their multirotor helicopter is derived and used around hover conditions.

The main contribution of this paper resides in the application of a generalized controllability analysis for the different configurations of multirotor unmanned aerial vehicles using small-time local controllability theory (STLC), in addition to the experimental validation of the obtained results of this study on a coaxial counter-rotating octorotor, by proposing and applying a system recovery strategy when the attitude of the octorotor was shown to be completely controllable, even after four motors failures.

The paper is organised as follows: in section 2, the dynamic model of a multirotor UAV is presented. In section 3, the controllability analysis is detailed then applied on different octorotor configurations, after multiple actuators failures, in order to compare their fault tolerance capabilities. In section 4, a control mixing and state feedback controller are applied to the 
coaxial octorotor in case of failures when the system is still proved to be controllable. The experimental validation is shown in section 5 .

\section{Dynamics Modelling}

Different configurations of octorotors exist. According to the arrangement and distribution of rotors, the most widely used layouts are:

- Coaxial octorotor: actuators aligned vertically but stacked in pairs so as to resemble a quadrotor as in Figure 1.

- Star-shaped octorotor: actuators aligned vertically and equally spaced around the vehicle. The rotor-turn directions can also be modified to obtain different configurations: PNPNPNPN octorotor or PPNNPPNN octorotor as in Figure 2 .

Despite the difference in type and configurations, octorotor dynamics in a hybrid coordinate system are given below where the vehicle's mass center translational dynamics are expressed in the inertial frame $R_{I}\left\{O_{I}, x_{I}, y_{I}, z_{I}\right\}$ and its angular dynamics are expressed in the body frame $R_{B}\left\{O_{B}, x_{B}, y_{B}, z_{B}\right\}$ :

$$
\begin{aligned}
& \ddot{x}=(\cos \phi \sin \theta \cos \psi+\sin \phi \sin \psi) * \frac{u_{f}}{m} \\
& \ddot{y}=(\cos \phi \sin \theta \sin \psi-\sin \phi \cos \psi) * \frac{u_{f}}{m} \\
& \ddot{z}=(\cos \phi \cos \theta) * \frac{u_{f}}{m}-g \\
& \dot{p}=\frac{I_{y y}-I_{z z}}{I_{x x}} q r-\frac{J_{r}}{I_{y x}} q \Omega+\frac{1}{I_{x x}} \tau_{\phi} \\
& \dot{q}=\frac{I_{z z}-I_{x x}}{I_{y y}} p r+\frac{J_{r}}{I_{y y}} p \Omega+\frac{1}{I_{y y}} \tau_{\theta} \\
& \dot{r}=\frac{I_{x x}-I_{y y}}{I_{z z}} p q+\frac{1}{I_{z z}} \tau_{\psi}
\end{aligned}
$$

$x, y$ and $z$ are the coordinates of the UAV center of mass in the inertial frame $R_{I} . m$ is the system mass and $I_{x x}, I_{y y}$ and $I_{z z}$ are the moments of inertia along $x_{B}, y_{B}$ and $z_{B}$ directions respectively. $\phi, \theta$ and $\psi$ are the roll, pitch and yaw Euler angles in the inertial frame $R_{I}$ and $p, q$ and $r$ are the angular velocities in the body-fixed frame $R_{B} . \tau_{\phi}, \tau_{\theta}$ and $\tau_{\psi}$ are the torques inputs around the $x_{B}, y_{B}$ and $z_{B}$ axes respectively. $J_{r}$ is the moment of inertia for each propeller and $\Omega$ is the overall residual propeller speed from the unbalanced rotor rotation.

$$
\Omega=\sum_{i=1}^{8} \omega_{i}
$$


where $\omega_{i}$, the $i^{\text {th }}$ propeller speed, is considered as positive or negative depending on the sense of rotation of the motor $i$. The standard definition of a positive rotation is used: this is defined as a counter-clockwise rotation around the axis as seen from directly in front of the axis line.

The rotating speed relation between the body coordinates and the inertial coordinates can be written as follows:

$$
\left[\begin{array}{c}
\dot{\phi} \\
\dot{\theta} \\
\dot{\psi}
\end{array}\right]=\left[\begin{array}{ccc}
1 & \sin \phi \tan \theta & \cos \phi \tan \theta \\
0 & \cos \phi & -\sin \phi \\
0 & \sin \phi / \cos \theta & \cos \phi / \cos \theta
\end{array}\right]\left[\begin{array}{c}
p \\
q \\
r
\end{array}\right]
$$

In case of small angles, this matrix is identical to the identity matrix $I_{3}$, and the following approximations can be used: $\dot{\phi}=p, \dot{\theta}=q$ and $\dot{\psi}=r$.

According to the geometry of the octorotor system, the mapping between the rotor lift and the total thrust and torques inputs is given by the effectiveness matrix $B$ :

$$
\left[\begin{array}{c}
u_{f} \\
\tau_{\phi} \\
\tau_{\theta} \\
\tau_{\psi}
\end{array}\right]=B *\left[\begin{array}{c}
F_{1} \\
F_{2} \\
\cdot \\
\cdot \\
F_{8}
\end{array}\right]
$$

where $F_{i}$ is the lift produced by the motor $i$.

For any p-rotor UAV, the control effectiveness matrix in parametrised form is given as:

$$
B=\left[\begin{array}{cccc}
\eta_{1} & \eta_{2} & \ldots & \eta_{p} \\
l_{1} \eta_{1} \sin \gamma_{1} & l_{2} \eta_{2} \sin \gamma_{2} & \ldots & l_{p} \eta_{p} \sin \gamma_{p} \\
-l_{1} \eta_{1} \cos \gamma_{1} & -l_{2} \eta_{2} \cos \gamma_{2} & \ldots & -l_{p} \eta_{p} \cos \gamma_{p} \\
\eta_{1} d_{1} & \eta_{2} d_{2} & \ldots & \eta_{p} d_{p}
\end{array}\right]
$$

The parameters $\eta_{i} \in[0,1]$ are used to account for rotor failure. The sign of $d_{i}$ depends on the direction of rotor rotation and $l_{i}$ is the length of the $i^{t h}$ arm. $\gamma_{i}$ is the angle subtended by the $i^{\text {th }}$ arm with the $x$-axis as shown in Figure 3. The rotor lift $F_{i}$ and torque $\tau_{i}$ produced by each motor in the $Z_{I}$ direction are given as:

$$
\begin{gathered}
F_{i}=K_{f} \omega_{i}^{2} \\
\tau_{i}=K_{t} \omega_{i}^{2}
\end{gathered}
$$

$K_{f}$ and $K_{t}$ are the thrust and reaction torques coefficients respectively. 

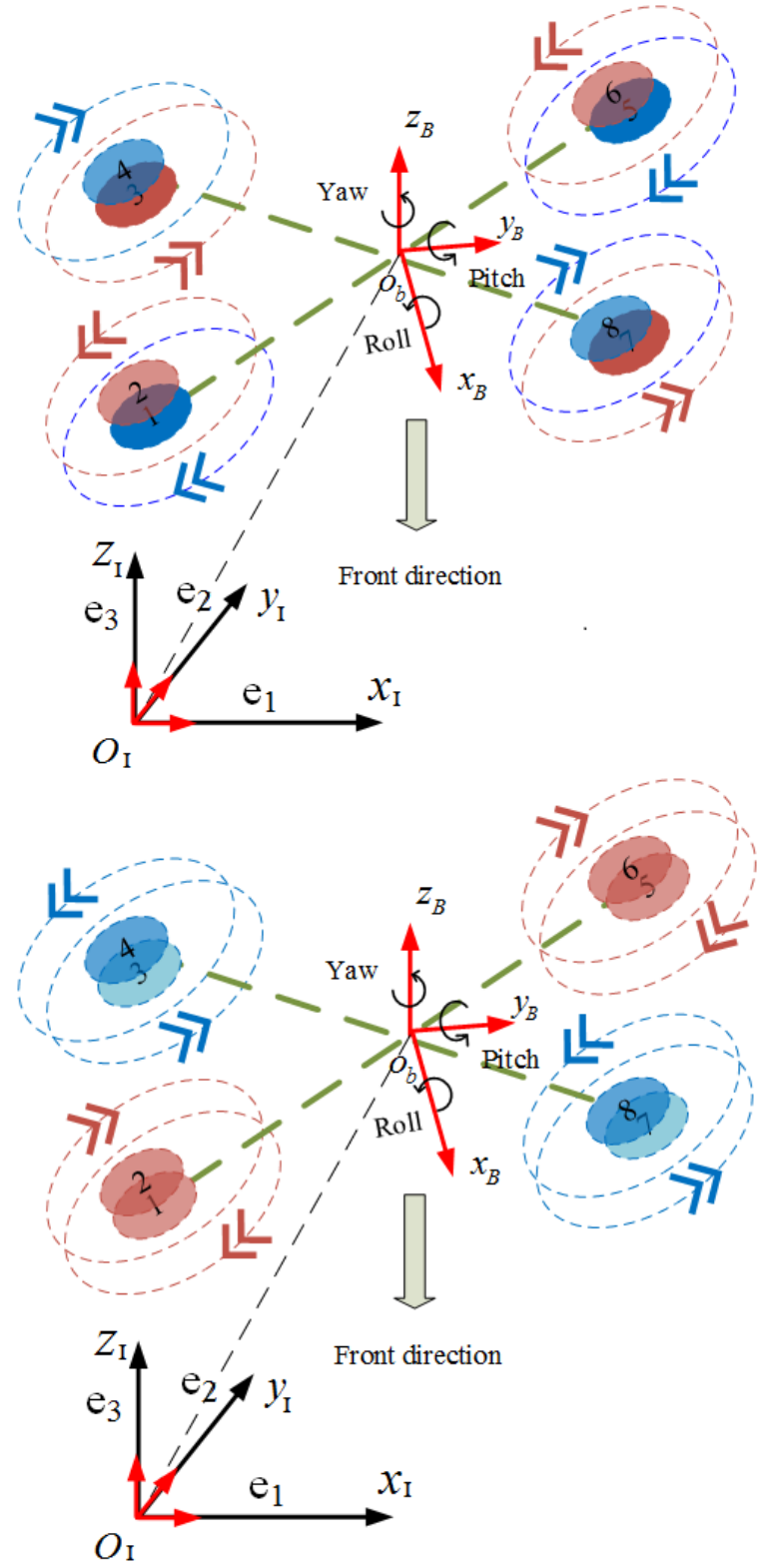

Figure 1: (a) The coaxial counter-rotating octorotor and (b) the coaxial co-rotating octorotor schematic representations 


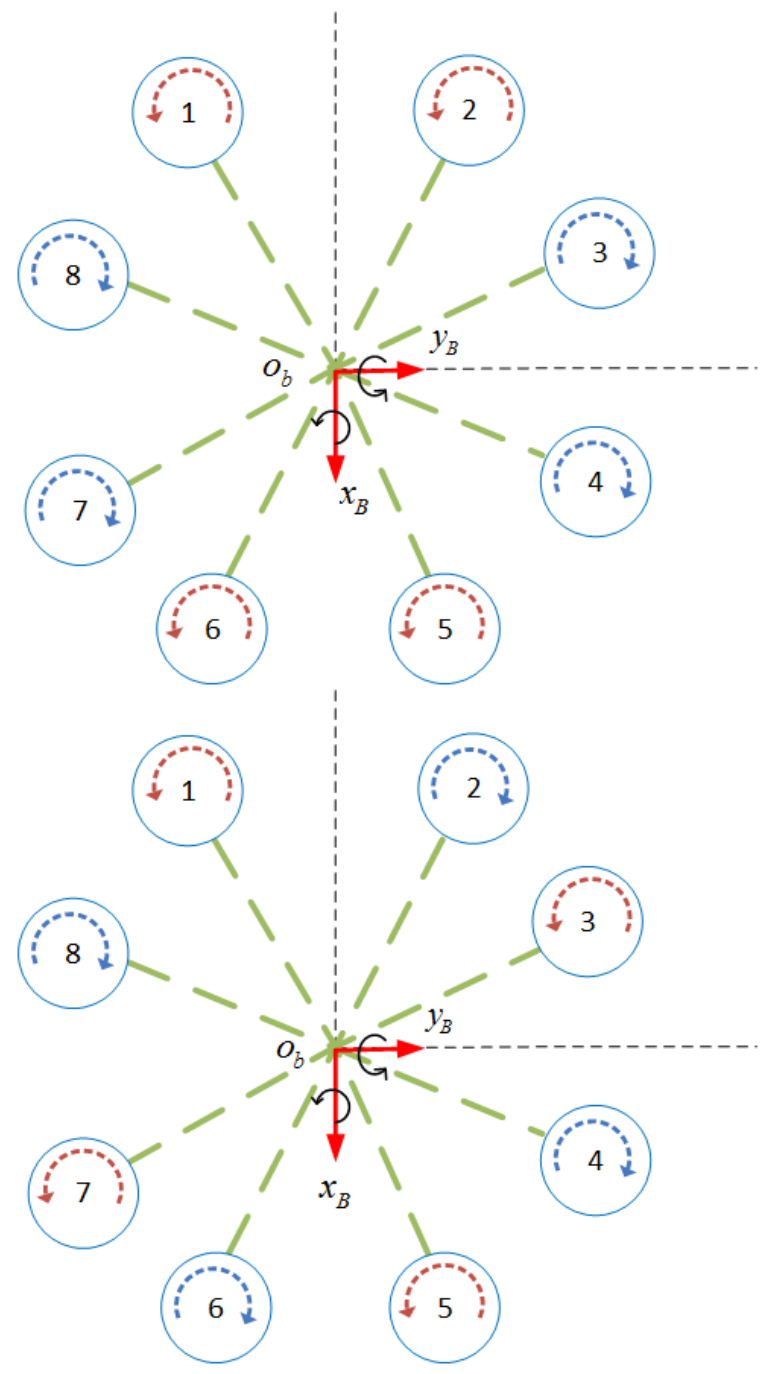

Figure 2: (a) The PPNNPPNN star-shaped octorotor and (b) the PNPNPNPN star-shaped octorotor schematic representations 


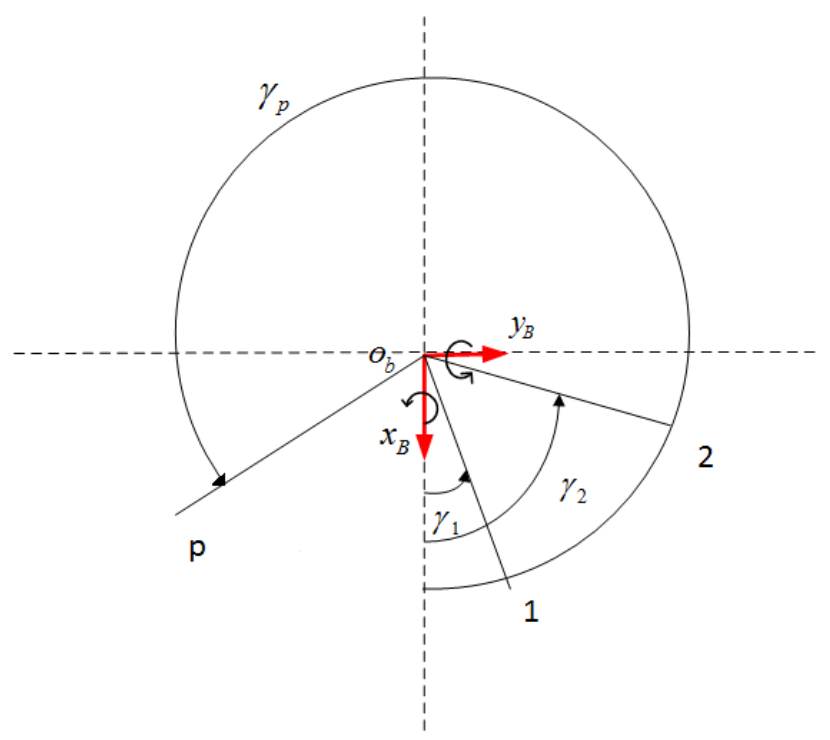

Figure 3: General p-rotor vehicle

For a coaxial octorotor, the combined thrust produced by two coaxial motors is given as:

$$
F_{i j}=\alpha_{i j} *\left(F_{i}+F_{j}\right) *\left(1+\frac{S_{s}}{S_{\text {prop }}}\right)
$$

$\alpha_{i j}$ is the coefficient of loss of aerodynamic efficiency due to the aerodynamic interference between the upper and lower rotors of each pair of coaxial rotors. $S=1+\frac{S_{s}}{S_{\text {prop }}}$ represents the shape factor of the propellers, with $S_{s}$ denoting the propellers surface and $S_{\text {prop }}$ the surface of the circle that the propeller would make when rotating.

\section{Controllability Analysis}

Before analysing the STLC of the multirotor attitude dynamics, some formal definitions and useful criteria need to be outlined. Let $M$ be an ndimensional analytic manifold, $U$ the set of admissible controls, and consider a general nonlinear control system written in control affine form as follows:

$$
\dot{x}=f(x, u)=f(x)+\sum_{i=1}^{m} g_{i}(x) u_{i} \quad(x \in M)
$$

where $f(x)$ is the drift vector field, and $g_{1}, g_{2}, \ldots, g_{m}$ are the control vector fields. The admissible control inputs $u_{i}$ are constrained to be non negative. 
Let $R\left(x_{0}, T\right)$ be the set of states $x$ such that there exists $u_{i}:[0, T] \rightarrow U$ that steers the control system from $x(0)$ to $x(T)=x_{f}$. We define $R\left(x_{0}, \leq\right.$ $T)=\cup_{0<t \leq T} R\left(x_{0}, T\right)$ to be the set of states reachable up to time $T$.

Definition 1. Goodwine \& Burdick (1996)] A system is said to be accessible from $x_{0}$ if there exists $\tau>0$ such that the interior of $R\left(x_{0}, \leq T\right)$ is not an empty set for $t \in] 0, \tau[$.

Definition 2. Goodwine \& Burdick (1996)] A system is said to be smalltime locally controllable $(S T L C)$ from $x_{0}$ if there exists $\tau>0$ such that $x_{0}$ lies in the interior of $R\left(x_{0}, \leq T\right)$ for all $\left.t \in\right] 0, \tau[$.

Let $L(\Delta)$ be the distribution of all independent vector fields that can be obtained by applying subsequent Lie bracket operations to the system vector fields $f, g_{1}, \ldots, g_{m}$, and let $\mathcal{L}$ be the accessibility distribution generated by $L(\Delta)$ :

$$
\mathcal{L}(x)=\operatorname{span}\{X(x): X \in L\}, \quad x \in M
$$

if $\operatorname{dim} \mathcal{L}\left(x_{0}\right)=\operatorname{dim} M$, then the system satisfies the Lie Algebra Rank Condition (LARC) at $x_{0}$ (J. M. Coron (2007)). If the control-affine system is driftless, the system is STLC if it verifies the LARC. However, with drift, this condition is not sufficient and the combinations of the vectors used to compose the Lie brackets of the Lie Algebra should be examined.

For a given Lie bracket $X$, consider the degree of a bracket with respect to a vector field $f$ or $g_{i}$, denoted by $\delta^{f}(X)$ and $\delta^{g_{i}}(X)$ respectively, to be the number of times that the superscripted vector field appears in the bracket $X$. We call a bracket $X$ bad if $\delta^{f}(X)+\sum_{i=1}^{m} \delta^{g_{i}}(X)$ is odd and $\sum_{i=1}^{m} \delta^{g_{i}}(X) \neq$ 1. Otherwise, the bracket is called good. Sussmann's General Theorem on Controllability (Sussmann (1987)) is reported below:

Theorem 1. [Sussmann (1987)] A system that satisfies the LARC by good Lie bracket terms up to degree $i$ is STLC if all bad Lie brackets of degree $j \leq i$ are neutralized.

A bad Lie bracket can be neutralized if it can be written as a linear combination of good Lie brackets of lower degree.

In the Sussmann's theorem, no constraints were considered on the control inputs and the control vector fields can be followed in both directions. The conditions for STLC for unilateral control inputs have been presented and 
generalized by Goodwine and Burdick in (Goodwine \& Burdick (1996)). The authors proposition, formally stated, is reported below:

Proposition 1. Goodwine \& Burdick (1996)] Assume that the system satisfies the LARC (condition 1) and that there exist coefficients $\lambda_{i}$ such that

$$
\sum_{i=1}^{m} \lambda_{i} g_{i}(x)=0 \quad \forall x \in \text { neighborhood of }\left(x_{0}\right)
$$

where $\lambda_{i} \in(0,1)$ (condition 2). Assume further that any bad bracket can be written as a linear combination of brackets of lower total degree (condition 3). Then the system is STLC at $x_{0}$.

The authors explained the intuition behind the restriction expressed in (10). None of the control inputs can be negative, however (10) can be solved for one $-g_{i}$ in terms of the other $g_{i}$ 's with positive coefficients and thus the corresponding $u_{i}$ acts as negative input.

\subsection{Controllability of an Octorotor}

The attitude dynamics of an octorotor can be expressed in control-affine form as in $(8)$, where $x=[\phi \dot{\phi} \theta \dot{\theta} \psi \dot{\psi}]^{T} \in M=R^{6}$ is the state. The control inputs $u_{i}$ are the thrusts $F_{i}$ provided by each motor. The drift field $f$ is written as:

$$
f=\left[\begin{array}{c}
\dot{\phi} \\
\frac{\left(I_{y y}-I_{z z}\right)}{I_{x x}} \dot{\theta} \dot{\psi} \\
\frac{\left(I_{z z}-I_{x x}\right)}{I_{y y}} \dot{\phi} \dot{\psi} \\
\psi \\
\frac{\left(I_{x x}-I_{y y}\right)}{I_{z z}} \dot{\phi} \dot{\theta}
\end{array}\right]
$$

\subsubsection{Coaxial Counter-Rotating Octorotor}

The control vector fields of a coaxial octorotor are: 


$$
\begin{aligned}
g_{1} & =\left[\begin{array}{llllll}
0 & -\frac{A}{I_{x x}} & 0 & -\frac{A}{I_{y y}} & 0 & -\frac{1}{I_{z z}} \frac{K_{t}}{K_{f}}
\end{array}\right]^{T} \\
g_{2} & =\left[\begin{array}{llllll}
0 & -\frac{A}{I_{x x}} & 0 & -\frac{A}{I_{y y}} & 0 & \frac{1}{I_{z z}} \frac{K_{t}}{K_{f}}
\end{array}\right]^{T} \\
g_{3} & =\left[\begin{array}{llllll}
0 & -\frac{A}{I_{x x}} & 0 & \frac{A}{I_{y y}} & 0 & \frac{1}{I_{z z}} \frac{K_{t}}{K_{f}}
\end{array}\right]^{T} \\
g_{4} & =\left[\begin{array}{llllll}
0 & -\frac{A}{I_{x x}} & 0 & \frac{A}{I_{y y}} & 0 & -\frac{1}{I_{z z}} \frac{K_{t}}{K_{f}}
\end{array}\right]^{T} \\
g_{5} & =\left[\begin{array}{llllll}
0 & \frac{A}{I_{x x}} & 0 & \frac{A}{I_{y y}} & 0 & -\frac{1}{I_{z z}} \frac{K_{t}}{K_{f}}
\end{array}\right]^{T} \\
g_{6} & =\left[\begin{array}{llllll}
0 & \frac{A}{I_{x x}} & 0 & \frac{A}{I_{y y}} & 0 & \frac{1}{I_{z z}} \frac{K_{t}}{K_{f}}
\end{array}\right]^{T} \\
g_{7} & =\left[\begin{array}{llllll}
0 & \frac{A}{I_{x x}} & 0 & -\frac{A}{I_{y y}} & 0 & \frac{1}{I_{z z}} \frac{K_{t}}{K_{f}}
\end{array}\right]^{T} \\
g_{8} & =\left[\begin{array}{llllll}
0 & \frac{A}{I_{x x}} & 0 & -\frac{A}{I_{y y}} & 0 & -\frac{1}{I_{z z}} \frac{K_{t}}{K_{f}}
\end{array}\right]^{T}
\end{aligned}
$$

with $A=l \frac{\sqrt{2}}{2}$. When applying the controllability test with unilateral control inputs, the Lie Algebra evaluates to:

$$
\mathcal{L}=\operatorname{span}\left\{g_{1}, g_{2}, g_{3},\left[f, g_{1}\right],\left[f, g_{2}\right],\left[f, g_{3}\right]\right\}
$$

with $\operatorname{det}(\mathcal{L})=\frac{-16 A^{4} K_{t}^{2}}{I_{x x}^{2} I_{y y}^{2} I_{z z}^{2} K_{f}^{2}} \neq 0$, so that its dimension is equal to 6 . The Lie Algebra is constructed using the Philip Hall basis, which is a sequence obtained through a breadth-first search that prunes all redundant vector fields arising from the skew symmetry and Jacobi identity properties. It is shown that three actuators are sufficient to ensure accessibility from equilibrium position provided that there is no duality between any two failed motors (two motors are called dual if they generate opposite torques in the three directions). However, intuitively, this will not imply that the system will be STLC with three actuators only. To prove that the system is STLC from zero-velocity states, we have to verify the three conditions of proposition 1 .

1. The LARC was verified with good Lie brackets of maximum degree 2, see Eq.(13);

2. We have from 12$) \sum_{i=1}^{8} g_{i}(x)=0$;

3. The only bad bracket with degree lower than or equal to 2 is $f$. However if we postulate that the octorotor is moving from an initial condition with velocity close to zero, then $f$ is neutralized;

Thus, the system is STLC from zero-velocity state. In this case, the LARC can be used directly to prove STLC since the control vectors are symmetric 
and then the system can move forward and backward.

\section{a- One motor failure:}

For one motor failure, the LARC can be verified as in (13) by any three control vectors corresponding to any three actuators, from the seven healthy ones, such that no duality exist between two of them. Again, the only bad Lie bracket that should be neutralized is $f$. Without loss of generality, we consider for example that motor 1 failed. Condition 2 is then verified by the following $\lambda_{i}$ 's: $\lambda_{2}=\lambda_{4}=\lambda_{8}=\alpha, \lambda_{3}=\lambda_{5}=\lambda_{7}=\frac{2}{3} \alpha, \lambda_{6}=\frac{1}{3} \alpha$ with $\alpha \in(0,1)$.

\section{b- Two motors failures:}

The results of the controllability evaluation for two motors failures are shown in Table I. Due to the system symmetry, and without loss of generality, the calculations are developed only for 5 cases, where motors 1 and $i$ fail, with $i=2,3, . .6$ (the following combinations are symmetric: $1 \& 3$ and $1 \& 7$ on one hand, $1 \& 4$ and $1 \& 8$ on the other hand). Conditions 1 and 3 are validated as demonstrated in the previous subsection. Condition 2 is verified for the different combinations using these $\lambda_{i} \mathrm{~s}$ :

C1: Motors $1 \& 2: \lambda_{5}=\lambda_{6}=0, \lambda_{3}=\lambda_{4}=\lambda_{7}=\lambda_{8}=\alpha$ with $\alpha \in(0,1)$;

C2: Motors $1 \& 3: \lambda_{2}=\lambda_{4}=\alpha, \lambda_{5}=\lambda_{6}=\lambda_{7}=\lambda_{8}=\frac{1}{2} \alpha$ with $\alpha \in(0,1)$;

C3: Motors $1 \& 4$ : $\lambda_{6}=\lambda_{7}=0, \lambda_{2}=\lambda_{3}=\lambda_{5}=\lambda_{8}=\alpha$ with $\alpha \in(0,1)$;

C4: Motors $1 \& 5: \lambda_{4}=\lambda_{8}=\alpha, \lambda_{2}=\lambda_{3}=\lambda_{6}=\lambda_{7}=\frac{1}{2} \alpha$ with $\alpha \in(0,1)$;

C5: Motors $1 \&$ 6: $\lambda_{i}=\alpha i=2, \ldots, 8$ with $\alpha \in(0,1)$;

Two special cases should be considered (C1 and C3), where some coefficients $\lambda$ are null, and so the system will be studied without considering the actuators corresponding to the zeros coefficients.

Case 3 (same procedure for case 1): Consider the system with only four actuators $(2,3,5$ and 8$)$. The $L(\Delta)$ is formed by considering the following Lie Brackets:

$$
\left\{g_{2}, g_{3},\left[f, g_{2}\right],\left[f, g_{3}\right],\left[g_{2},\left[f, g_{5}\right]\right],\left[f,\left[f, g_{2}\right]\right]\right\}
$$


$\operatorname{det}(\mathcal{L})=\frac{\left(8 d^{3} K_{t}^{3} *\left(I_{y y}-I_{z z}\right) *\left(I_{y y}^{2} K_{t} \dot{\theta}+I_{z z}^{2} A K_{f} \dot{\psi}-I_{y y} I_{z z} K_{t} \dot{\theta}-I_{y y} I_{z z} A K_{f} \dot{\psi}\right)\right)}{\left(I_{x x}^{2} I_{y y}^{4} I_{z z}^{4} K_{f}^{4}\right)} \neq 0$ if $\dot{\theta} \neq 0$ or $\dot{\psi} \neq 0$. The system is found to be accessible for all conditions except when $\dot{\theta}=0$ or $\dot{\psi}=0$. The LARC is not verified at the equilibrium position and the system is not STLC from zero velocities states.

\begin{tabular}{|c|c|c|c|}
\hline Motors failures & Controllability & Motors failures & Controllability \\
\hline $1 \& 2$ & Inaccessible from zero velocities & $1 \& 3$ & STLC \\
\hline $1 \& 4$ & Inaccessible from zero velocities & $1 \& 5$ & STLC \\
\hline $1 \& 6$ & STLC & $1 \& 7$ & STLC \\
\hline $1 \& 8$ & Inaccessible from zero velocities & $2 \& 3$ & Inaccessible from zero velocities \\
\hline $2 \& 4$ & STLC & $2 \& 5$ & STLC \\
\hline $2 \& 6$ & STLC & $2 \& 7$ & Inaccessible from zero velocities \\
\hline $2 \& 8$ & STLC & $3 \& 4$ & Inaccessible from zero velocities \\
\hline $3 \& 5$ & STLC & $3 \& 6$ & Inaccessible from zero velocities \\
\hline $3 \& 7$ & STLC & $3 \& 8$ & STLC \\
\hline $4 \& 5$ & Inaccessible from zero velocities & $4 \& 6$ & STLC \\
\hline $4 \& 7$ & STLC & $4 \& 8$ & STLC \\
\hline $5 \& 6$ & Inaccessible from zero velocities & $5 \& 7$ & STLC \\
\hline $5 \& 8$ & Inaccessible from zero velocities & $6 \& 7$ & Inaccessible from zero velocities \\
\hline $6 \& 8$ & STLC & $7 \& 8$ & Inaccessible from zero velocities \\
\hline
\end{tabular}

Table 1: STLC analysis for the coaxial counter-rotating octorotor after two motors failures

Note that, in this table, acessibility is not equivalent to small time local controllability. However, the inaccessibility from zero velocity states imply that the system is not small time locally controllable at the equilibrium given the set of sufficient conditions used.

In 12 from the 28 combinations, the octorotor is not STLC from zero-velocity states. The results are extended for a higher number of rotors failures (three and four). It can be deduced that the octorotor is STLC just for 16 from 56 combinations of three motors failures (any three upper motors failures or any three lower motors failures) and for just 2 combinations of four motors failures: the four upper or the four lower motors.

The results obtained above representing the controllability status from the equilibrium positions are the same of those obtained from the application of the Attainable Control Set method as in (Schneider et al. (2012)). However, the latter method is used for the study of static controllability, which is based on the computation of the control authority and the attainable control, while neglecting the nonlinear dynamics of the multirotor.

\section{c- Stabilizability analysis}

We show that the system is not even stabilizable by a continuous statefeedback control law in the cases where it is not small-time local controllable. A necessary condition (Brockett 1983) for the existence of a continuous state 
feedback law that asymptotically stabilizes this system is that the image of the mapping $R^{6} \mathrm{x} R^{m} \rightarrow R^{6}$ defined by:

$$
\left(x, u_{1}, u_{2}, \ldots, u_{m}\right) \rightarrow f(x)+\sum_{i=1}^{m} g_{i}(x) u_{i}
$$

contains a neighbourhood of zero. This condition is satisfied if and only if the system

$$
\left(\begin{array}{c}
\epsilon_{1} \\
\epsilon_{2} \\
\epsilon_{3} \\
\epsilon_{4} \\
\epsilon_{5} \\
\epsilon_{6}
\end{array}\right)=\left(\begin{array}{c}
\dot{\phi} \\
\frac{I_{y y}-I_{z z}}{I_{x x}} \dot{\theta} \dot{\psi} \\
\dot{\psi} \\
\frac{I_{z z}-I_{x x}}{I_{y y}} \dot{\phi} \dot{\psi} \\
\dot{\psi} \\
\frac{I_{x x}-I_{y y}}{I_{z z}} \dot{\phi} \dot{\theta}
\end{array}\right)+\left(\begin{array}{c}
0 \\
-\frac{A}{I_{x x}} \\
0 \\
-\frac{A}{I_{y y}} \\
0 \\
-\frac{1}{I_{z z}} \frac{K_{t}}{K_{f}}
\end{array}\right) u_{1}+\left(\begin{array}{c}
0 \\
-\frac{A}{I_{x x}} \\
0 \\
-\frac{A}{I_{y y}} \\
0 \\
\frac{1}{I_{z z}} \frac{K_{t}}{K_{f}}
\end{array}\right) u_{2}+\ldots+\left(\begin{array}{c}
0 \\
\frac{A}{I_{x x}} \\
0 \\
-\frac{A}{I_{y y}} \\
0 \\
-\frac{1}{I_{z z}} \frac{K_{t}}{K_{f}}
\end{array}\right) u_{8}
$$

is solvable for any $\epsilon=\left(\epsilon_{1} \epsilon_{2} \epsilon_{3} \epsilon_{4} \epsilon_{5} \epsilon_{6}\right)$ near 0 . Let $\epsilon_{1}=\epsilon_{3}=\epsilon_{5}=0$. This implies $\dot{\phi}=\dot{\theta}=\dot{\psi}=0, \frac{I_{y y}-I_{z z}}{I_{x x}} \dot{\theta} \dot{\psi}=\frac{I_{z z}-I_{x x}}{I_{y y}} \dot{\phi} \dot{\psi}=\frac{I_{x x}-I_{y y}}{I_{z z}} \dot{\phi} \dot{\theta}=0$ and 16 becomes:

$$
\left(\begin{array}{c}
\epsilon_{2} \\
\epsilon_{4} \\
\epsilon_{6}
\end{array}\right)=\left(\begin{array}{cccccccc}
-\frac{A}{I_{x x}} & -\frac{A}{I_{x x}} & -\frac{A}{I_{x x}} & -\frac{A}{I_{x x}} & \frac{A}{I_{x x}} & \frac{A}{I_{x x}} & \frac{A}{I_{x x}} & \frac{A}{I_{x x}} \\
-\frac{A}{I_{y y}} & -\frac{A}{I_{y y}} & \frac{A}{I_{y y}} & \frac{A}{I_{y y}} & \frac{A}{I_{y y}} & \frac{A}{I_{y y}} & -\frac{A}{I_{y y}} & -\frac{A}{I_{y y}} \\
-\frac{1}{I_{z z}} \frac{K_{t}}{K_{f}} & \frac{1}{I_{z z}} \frac{K_{t}}{K_{f}} & \frac{1}{I_{z z}} \frac{K_{t}}{K_{f}} & -\frac{1}{I_{z z}} \frac{K_{t}}{K_{f}} & -\frac{1}{I_{z z}} \frac{K_{t}}{K_{f}} & \frac{1}{I_{z z}} \frac{K_{t}}{K_{f}} & \frac{1}{I_{z z}} \frac{K_{t}}{K_{f}} & -\frac{1}{I_{z z}} \frac{K_{t}}{K_{f}}
\end{array}\right)\left(\begin{array}{c}
u_{1} \\
u_{2} \\
u_{3} \\
u_{4} \\
u_{5} \\
u_{6} \\
u_{7} \\
u_{8}
\end{array}\right)
$$

For failures of motors $1 \& 2$, no points of the form $\epsilon=(0,0,0, \delta, 0,0), \delta<0$, are in the image of the mapping. Thus, the condition is violated. Same calculations and conclusions are conducted for the cases where the system was shown not to be controllable.

\section{d- Controllability without yaw angle and angular velocity}

Considering that we forsake control of the yaw angle and the angular velocity in critical cases, we study in this section the controllability of the system attitude including roll and pitch states. We will consider only the two cases $\mathrm{C}_{1}$ and $\mathrm{C}_{3}$. 
Consider $x=[\phi \dot{\phi} \theta \dot{\theta}]^{T} \in R^{4}$ as the attitude state vector. $f$ and $g_{i}$ are then written as:

$$
\begin{aligned}
& f=\left[\begin{array}{c}
\dot{\phi} \\
\frac{\left(I_{y y}-I_{z z}\right)}{I_{x x}} \dot{\theta} \dot{\psi} \\
\dot{\theta} \\
\frac{\left(I_{z z}-I_{x x}\right)}{I_{y y}} \dot{\phi} \dot{\psi}
\end{array}\right] \\
& g_{1}=\left[\begin{array}{llll}
0 & -\frac{A}{I_{x x}} & 0 & -\frac{A}{I_{y y}}
\end{array}\right]^{T} \quad g_{2}=\left[\begin{array}{llll}
0 & -\frac{A}{I_{x x}} & 0 & -\frac{A}{I_{y y}}
\end{array}\right]^{T} \\
& g_{3}=\left[\begin{array}{llll}
0 & -\frac{A}{I_{x x}} & 0 & \frac{A}{I_{y y}}
\end{array}\right]^{T} \quad g_{4}=\left[\begin{array}{llll}
0 & -\frac{A}{I_{x x}} & 0 & \frac{A}{I_{y y}}
\end{array}\right]^{T} \\
& g_{5}=\left[\begin{array}{cccc}
0 & \frac{A}{I_{x x}} & 0 & \frac{A}{I_{y y}}
\end{array}\right]^{T} \quad g_{6}=\left[\begin{array}{llll}
0 & \frac{A}{I_{x x}} & 0 & \frac{A}{I_{y y}}
\end{array}\right]^{T} \\
& g_{7}=\left[\begin{array}{llll}
0 & \frac{A}{I_{x x}} & 0 & -\frac{A}{I_{y y}}
\end{array}\right]^{T} \quad g_{8}=\left[\begin{array}{llll}
0 & \frac{A}{I_{x x}} & 0 & -\frac{A}{I_{y y}}
\end{array}\right]^{T}
\end{aligned}
$$

In case of failures of motors $1 \& 4$, the Lie Algebra evaluates to:

$$
\mathcal{L}=\operatorname{span}\left\{g_{2}, g_{3},\left[f, g_{2}\right],\left[f, g_{3}\right]\right\}
$$

with $\operatorname{det}(\mathcal{L})=-\frac{4 A^{2}}{I_{x x}^{2} * I_{y y}^{2}} \neq 0$ for Lie brackets of maximum degree of 2 . Having $f=0$ at zero velocities states, and $\sum g_{i}(x) u_{i}=0, i=2,3,5,8$, the LARC guarantees that the system is STLC from the equilibrium when sacrificing the yaw control.

In case of failures of motors $1 \& 2$, consider the reduced system composed of the roll, pitch and yaw rates only. The Lie Algebra is:

$$
\mathcal{L}=\operatorname{span}\left\{g_{3},\left[f, g_{3}\right],\left[f,\left[f, g_{3}\right]\right]\right\}
$$

$\operatorname{det}(\mathcal{L}) \neq 0$ if $\dot{\psi} \neq 0$ or $\dot{\psi} \neq 0 \& \dot{\phi} \neq 0$ or $\dot{\psi} \neq 0 \& \dot{\theta} \neq 0$. Thus, the reduced attitude can be controlled if the system rotates around the $\mathrm{z}$ axis in a single direction since $\dot{\psi}$ can not be null. The difference between these two cases is that the yaw state is not controlled when the motors $1 \& 4$ fail but the yaw rate can be null. However, the octorotor must rotate continuously around the $\mathrm{z}$ axis in the other case.

\subsubsection{Coaxial Co-Rotating Octorotor}

The configuration of the coaxial co-rotating octorotor is shown in Fig. 1.b.

The attitude system is also written as:

$$
\dot{x}=f_{1}(x)+\sum_{i=1}^{8} g_{1_{i}}(x) u_{i}
$$


where $f_{1}(x)=f(x)$ and $g_{1_{i}}$ are as follows:

$$
\begin{aligned}
g_{1_{1}} & =\left[\begin{array}{llllll}
0 & -\frac{A}{I_{x x}} & 0 & -\frac{A}{I_{y y}} & 0 & -\frac{1}{I_{z z}} \frac{K_{t}}{K_{f}}
\end{array}\right]^{T} \\
g_{1_{2}} & =\left[\begin{array}{llllll}
0 & -\frac{A}{I_{x x}} & 0 & -\frac{A}{I_{y y}} & 0 & -\frac{1}{I_{z z}} \frac{K_{t}}{K_{f}}
\end{array}\right]^{T} \\
g_{1_{3}} & =\left[\begin{array}{llllll}
0 & -\frac{A}{I_{x x}} & 0 & \frac{A}{I_{y y}} & 0 & \frac{1}{I_{z z}} \frac{K_{t}}{K_{f}}
\end{array}\right]^{T} \\
g_{1_{4}} & =\left[\begin{array}{llllll}
0 & -\frac{A}{I_{x x}} & 0 & \frac{A}{I_{y y}} & 0 & \frac{1}{I_{z z}} \frac{K_{t}}{K_{f}}
\end{array}\right]^{T} \\
g_{1_{5}} & =\left[\begin{array}{llllll}
0 & \frac{A}{I_{x x}} & 0 & \frac{A}{I_{y y}} & 0 & -\frac{1}{I_{z z}} \frac{K_{t}}{K_{f}}
\end{array}\right]^{T} \\
g_{1_{6}} & =\left[\begin{array}{llllll}
0 & \frac{A}{I_{x x}} & 0 & \frac{A}{I_{y y}} & 0 & -\frac{1}{I_{z z}} \frac{K_{t}}{K_{f}}
\end{array}\right]^{T} \\
g_{1_{7}} & =\left[\begin{array}{llllll}
0 & \frac{A}{I_{x x}} & 0 & -\frac{A}{I_{y y}} & 0 & \frac{1}{I_{z z}} \frac{K_{t}}{K_{f}}
\end{array}\right]^{T} \\
g_{1_{8}} & =\left[\begin{array}{llllll}
0 & \frac{A}{I_{x x}} & 0 & -\frac{A}{I_{y y}} & 0 & \frac{1}{I_{z z}} \frac{K_{t}}{K_{f}}
\end{array}\right]^{T}
\end{aligned}
$$

\begin{tabular}{|c|c|c|c|}
\hline Motors failures & Controllability & Motors failures & Controllability \\
\hline $1 \& 2 \& 3$ & Inaccessible from zero velocities & $1 \& 2 \& 4$ & Inaccessible from zero velocities \\
\hline $1 \& 2 \& 5$ & Inaccessible from zero velocities & $1 \& 2 \& 6$ & Inaccessible from zero velocities \\
\hline $1 \& 2 \& 7$ & Inaccessible from zero velocities & $1 \& 2 \& 7$ & Inaccessible from zero velocities \\
\hline $1 \& 3 \& 4$ & Inaccessible from zero velocities & $1 \& 3 \& 5$ & STLC \\
\hline $1 \& 3 \& 6$ & STLC & $1 \& 3 \& 7$ & STLC \\
\hline $1 \& 3 \& 8$ & STLC & $1 \& 4 \& 5$ & STLC \\
\hline $1 \& 4 \& 6$ & STLC & $1 \& 4 \& 7$ & STLC \\
\hline $1 \& 4 \& 8$ & STLC & $1 \& 5 \& 6$ & STLC \\
\hline $1 \& 5 \& 7$ & STLC & $1 \& 5 \& 8$ & STLC \\
\hline $1 \& 6 \& 7$ & STLC & $1 \& 6 \& 8$ & STLC \\
\hline $1 \& 7 \& 8$ & Inaccessible from zero velocities & & \\
\hline
\end{tabular}

The same procedure as in section 4.1.1 is followed for this configuration.

\begin{tabular}{|c|c|c|c|}
\hline Motors failures & Controllability & Motors failures & Controllability \\
\hline $1 \& 2 \&-\&-$ & Inaccessible from zero velocities & $1 \& 3 \& 4 \&-$ & Inaccessible from zero velocities \\
\hline $1 \& 3 \& 5 \& 6$ & Inaccessible from zero velocities & $1 \& 3 \& 5 \& 7$ & STLC \\
\hline $1 \& 3 \& 5 \& 8$ & STLC & $1 \& 3 \& 6 \& 7$ & STLC \\
\hline $1 \& 3 \& 6 \& 8$ & STLC & $1 \& 3 \& 7 \& 8$ & Inaccessible from zero velocities \\
\hline $1 \& 4 \& 5 \& 6$ & Inaccessible from zero velocities & $1 \& 4 \& 5 \& 7$ & STLC \\
\hline $1 \& 4 \& 5 \& 8$ & STLC & $1 \& 4 \& 6 \& 7$ & STLC \\
\hline $1 \& 4 \& 6 \& 8$ & STLC & $1 \& 4 \& 7 \& 8$ & Inaccessible from zero velocities \\
\hline $1 \& 5 \& 6 \& 7$ & STLC & $1 \& 5 \& 6 \& 8$ & STLC \\
\hline $1 \& 5 \& 7 \& 8$ & Inaccessible from zero velocities & $1 \& 6 \& 7 \& 8$ & Inaccessible from zero velocities \\
\hline
\end{tabular}
Calculations details are omitted due to the lack in space. The small time local controllability results after two, three and four motors failures are shown in Tables 2, 3 and 4 respectively.

\begin{tabular}{c|c||c|c}
\hline Motors failures & Controllability & Motors failures & Controllability \\
\hline $1 \& 2$ & Inaccessible from zero velocities & $1 \& 3$ & STLC \\
$1 \& 4$ & STLC & $1 \& 5$ & STLC \\
$1 \& 6$ & STLC & $1 \& 7$ & STLC \\
$1 \& 8$ & STLC & STLC \\
$2 \& 4$ & STLC & $2 \& 5$ & STLC \\
$2 \& 6$ & STLC & $3 \& 4$ & STLC \\
$2 \& 8$ & STLC & $3 \& 6$ & Inaccessible from zero velocities \\
$3 \& 5$ & STLC & $3 \& 8$ & STLC \\
$3 \& 7$ & STLC & $4 \& 6$ & STLC \\
$4 \& 5$ & STLC & $4 \& 8$ & STLC \\
$4 \& 7$ & STLC & $5 \& 7$ & STLC \\
$5 \& 6$ & STLC & $6 \& 7$ & STLC \\
$5 \& 8$ & STLC & S \& 8 & Inaccessible from zero velocities \\
68 & STLC &
\end{tabular}

Table 2: STLC analysis of the coaxial co-rotating octorotor after two motors failures.

Table 3: STLC analysis of the coaxial co-rotating octorotor after three motors failures. 
Table 4: STLC analysis of the coaxial co-rotating octorotor after four motors failures.

\subsubsection{PPNNPPNN Star-Shaped Octorotor}

For this configuration (see Fig. 2,a), the differential equations describing the star-shaped octorotor dynamics are the same of those for coaxial octorotor. However, the effectiveness matrix differs since the distribution of the rotors is not the same.

Thus $f_{2}=f$ and $g_{2_{i}}$ are written as:

$$
\begin{aligned}
& g_{2_{1}}=\left[\begin{array}{llllll}
0 & \frac{1}{I_{x x}} K_{f} l \sin \left(\gamma_{1}\right) & 0 & -\frac{1}{I_{y y}} K_{f} l \cos \left(\gamma_{1}\right) & 0 & \frac{1}{I_{z z}} \frac{K_{t}}{K_{f}}
\end{array}\right]^{T}
\end{aligned}
$$

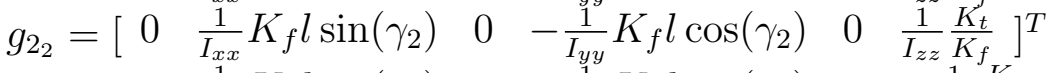

$$
\begin{aligned}
& g_{2_{3}}=\left[\begin{array}{llllll}
0 & \frac{1}{I_{x x}} K_{f} l \sin \left(\gamma_{3}\right) & 0 & -\frac{1}{I_{y y}} K_{f} l \cos \left(\gamma_{3}\right) & 0 & -\frac{1}{I_{z z}} \frac{K_{t}}{K_{f}}
\end{array}\right]^{T} \\
& g_{2_{4}}=\left[\begin{array}{llllll}
0 & \frac{1}{I_{x x}} K_{f} l \sin \left(\gamma_{4}\right) & 0 & -\frac{1}{I_{y y}} K_{f} l \cos \left(\gamma_{4}\right) & 0 & -\frac{1}{I_{z z}} \frac{K_{t}}{K_{f}}
\end{array}\right]^{T} \\
& g_{2_{5}}=\left[\begin{array}{llllll}
0 & \frac{1}{I_{x x}} K_{f} l \sin \left(\gamma_{5}\right) & 0 & -\frac{1}{I_{y y}} K_{f} l \cos \left(\gamma_{5}\right) & 0 & \frac{1}{I_{z z}} \frac{K_{t}}{K_{f}}
\end{array}\right]^{T} \\
& g_{2_{6}}=\left[\begin{array}{llllll}
0 & \frac{1}{I_{x x}} K_{f} l \sin \left(\gamma_{6}\right) & 0 & -\frac{1}{I_{y y}} K_{f} l \cos \left(\gamma_{6}\right) & 0 & \frac{1}{I_{z z}} \frac{K_{t}}{K_{f}}
\end{array}\right]^{T} \\
& g_{2_{7}}=\left[\begin{array}{llllll}
0 & \frac{1}{I_{x x}} K_{f} l \sin \left(\gamma_{7}\right) & 0 & -\frac{1}{I_{y y}} K_{f} l \cos \left(\gamma_{7}\right) & 0 & -\frac{1}{I_{z z}} \frac{K_{t}}{K_{f}}
\end{array}\right]^{T} \\
& g_{2_{8}}=\left[\begin{array}{llllll}
0 & \frac{1}{I_{x x}} K_{f} l \sin \left(\gamma_{8}\right) & 0 & -\frac{1}{I_{y y}} K_{f} l \cos \left(\gamma_{8}\right) & 0 & -\frac{1}{I_{z z}} \frac{K_{t}}{K_{f}}
\end{array}\right]^{T}
\end{aligned}
$$

where $\gamma_{i}$ denotes the angle between the arms of the vehicle and the major $x$-axis as shown previously in Fig. 3 .

\begin{tabular}{|c|c|c|c|}
\hline Motors failures & Controllability & Motors failures & Controllability \\
\hline $1 \& 2 \& 3$ & Inaccessible from zero velocities & $1 \& 2 \& 4$ & Inaccessible from zero velocities \\
\hline $1 \& 2 \& 5$ & Inaccessible from zero velocities & $1 \& 2 \& 6$ & Inaccessible from zero velocities \\
\hline $1 \& 2 \& 7$ & Inaccessible from zero velocities & $1 \& 2 \& 7$ & Inaccessible from zero velocities \\
\hline $1 \& 3 \& 4$ & Inaccessible from zero velocities & $1 \& 3 \& 5$ & STLC \\
\hline $1 \& 3 \& 6$ & STLC & $1 \& 3 \& 7$ & STLC \\
\hline $1 \& 3 \& 8$ & STLC & $1 \& 4 \& 5$ & STLC \\
\hline $1 \& 4 \& 6$ & STLC & $1 \& 4 \& 7$ & STLC \\
\hline $1 \& 4 \& 8$ & STLC & $1 \& 5 \& 6$ & STLC \\
\hline $1 \& 5 \& 7$ & STLC & $1 \& 5 \& 8$ & STLC \\
\hline $1 \& 6 \& 7$ & STLC & $1 \& 6 \& 8$ & STLC \\
\hline $1 \& 7 \& 8$ & Inaccessible from zero velocities & & \\
\hline
\end{tabular}

The controllability results are also presented after two, three and four motors failures in Tables 5, 6 and 7 respectively, and will be discussed later.

\begin{tabular}{c|c||c|c}
\hline Motors failures & Controllability & Motors failures & Controllability \\
\hline $1 \& 2$ & Inaccessible from zero velocities & $1 \& 3$ & STLC \\
$1 \& 4$ & STLC & $1 \& 5$ & STLC \\
$1 \& 6$ & STLC & $1 \& 7$ & STLC \\
$1 \& 8$ & STLC & 23 & STLC \\
$2 \& 4$ & STLC & $2 \& 7$ & STLC \\
$2 \& 6$ & STLC & $3 \& 4$ & Inaccessible from zero velocities \\
$2 \& 8$ & STLC & $3 \& 6$ & STLC \\
$3 \& 5$ & STLC & $3 \& 8$ & STLC \\
$3 \& 7$ & STLC & $4 \& 6$ & STLC \\
$4 \& 5$ & STLC & $4 \& 8$ & STLC \\
$4 \& 7$ & STLC & $5 \& 7$ & STLC \\
$5 \& 6$ & Inaccessible from zero velocities & $6 \& 7$ & STLC \\
$5 \& 8$ & STLC & $7 \& 8$ & Inaccessible from zero velocities \\
$6 \& 8$ & STLC &
\end{tabular}

Table 5: STLC analysis of the PPNNPPNN star-shaped octorotor after two motors failures. 
Table 6: STLC analysis of the PPNNPPNN star-shaped octorotor after three motors failures.

\begin{tabular}{|c|c|c|c|}
\hline Motors failures & Controllability & Motors failures & Controllability \\
\hline $1 \& 2 \&-\&-$ & Inaccessible from zero velocities & $1 \& 3 \& 4 \&-$ & Inaccessible from zero velocities \\
\hline $1 \& 3 \& 5 \& 6$ & Inaccessible from zero velocities & $1 \& 3 \& 5 \& 7$ & STLC \\
\hline $1 \& 3 \& 5 \& 8$ & STLC & $1 \& 3 \& 6 \& 7$ & STLC \\
\hline $1 \& 3 \& 6 \& 8$ & STLC & $1 \& 3 \& 7 \& 8$ & Inaccessible from zero velocities \\
\hline $1 \& 4 \& 5 \& 6$ & Inaccessible from zero velocities & $1 \& 4 \& 5 \& 7$ & STLC \\
\hline $1 \& 4 \& 5 \& 8$ & STLC & $1 \& 4 \& 6 \& 7$ & STLC \\
\hline $1 \& 4 \& 6 \& 8$ & STLC & $1 \& 4 \& 7 \& 8$ & Inaccessible from zero velocities \\
\hline $1 \& 5 \& 6 \& 7$ & Inaccessible from zero velocities & $1 \& 5 \& 6 \& 8$ & Inaccessible from zero velocities \\
\hline $1 \& 5 \& 7 \& 8$ & Inaccessible from zero velocities & $1 \& 6 \& 7 \& 8$ & Inaccessible from zero velocities \\
\hline
\end{tabular}

Table 7: STLC analysis of the PPNNPPNN star-shaped octorotor after four motors failures.

\subsubsection{PNPNPNPN Star-Shaped Octorotor}

The control vector inputs of this configuration (see Fig. 2.b) are written as:

$$
\begin{aligned}
& g_{2_{1}}=\left[\begin{array}{llllll}
0 & \frac{1}{I_{x x}} K_{f} l \sin \left(\gamma_{1}\right) & 0 & -\frac{1}{I_{y y}} K_{f} l \cos \left(\gamma_{1}\right) & 0 & \frac{1}{I_{z z}} \frac{K_{t}}{K_{f}}
\end{array}\right]^{T}
\end{aligned}
$$

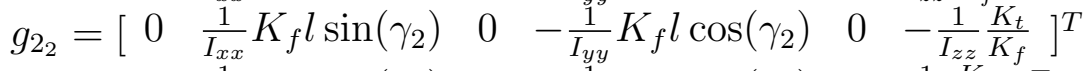

$$
\begin{aligned}
& g_{2_{3}}=\left[\begin{array}{llllll}
0 & \frac{1}{I_{x x}} K_{f} l \sin \left(\gamma_{3}\right) & 0 & -\frac{1}{I_{y y}} K_{f} l \cos \left(\gamma_{3}\right) & 0 & \frac{1}{I_{z z}} \frac{K_{t}}{K_{f}}
\end{array}\right]^{T} \\
& g_{2_{4}}=\left[\begin{array}{llllll}
0 & \frac{1}{I_{x x}} K_{f} l \sin \left(\gamma_{4}\right) & 0 & -\frac{1}{I_{y y}} K_{f} l \cos \left(\gamma_{4}\right) & 0 & -\frac{1}{I_{z z}} \frac{K_{t}}{K_{f}}
\end{array}\right]^{T} \\
& g_{2_{5}}=\left[\begin{array}{llllll}
0 & \frac{1}{I_{x x}} K_{f} l \sin \left(\gamma_{5}\right) & 0 & -\frac{1}{I_{y y}} K_{f} l \cos \left(\gamma_{5}\right) & 0 & \frac{1}{I_{z z}} \frac{K_{t}}{K_{f}}
\end{array}\right]^{T} \\
& g_{2_{6}}=\left[\begin{array}{llllll}
0 & \frac{1}{I_{x x}} K_{f} l \sin \left(\gamma_{6}\right) & 0 & -\frac{1}{I_{y y}} K_{f} l \cos \left(\gamma_{6}\right) & 0 & -\frac{1}{I_{z z}} \frac{K_{t}}{K_{f}}
\end{array}\right]^{T} \\
& g_{2_{7}}=\left[\begin{array}{llllll}
0 & \frac{1}{I_{x x}} K_{f} l \sin \left(\gamma_{7}\right) & 0 & -\frac{1}{I_{y y}} K_{f} l \cos \left(\gamma_{7}\right) & 0 & \frac{1}{I_{z z}} \frac{K_{t}}{K_{f}}
\end{array}\right]^{T} \\
& g_{2_{8}}=\left[\begin{array}{llllll}
0 & \frac{1}{I_{x x}} K_{f} l \sin \left(\gamma_{8}\right) & 0 & -\frac{1}{I_{y y}} K_{f} l \cos \left(\gamma_{8}\right) & 0 & -\frac{1}{I_{z z}} \frac{K_{t}}{K_{f}}
\end{array}\right]^{T}
\end{aligned}
$$

\begin{tabular}{|c|c|c|c|}
\hline Motors failures & Controllability & Motors failures & Controllability \\
\hline $1 \& 2 \& 3$ & Inaccessible from zero velocities & $1 \& 2 \& 4$ & STLC \\
\hline $1 \& 2 \& 5$ & STLC & $1 \& 2 \& 6$ & STLC \\
\hline $1 \& 2 \& 7$ & STLC & $1 \& 2 \& 8$ & Inaccessible from zero velocities \\
\hline $1 \& 3 \& 4$ & STLC & $1 \& 3 \& 5$ & Inaccessible from zero velocities \\
\hline $1 \& 3 \& 6$ & STLC & $1 \& 3 \& 7$ & Inaccessible from zero velocities \\
\hline $1 \& 3 \& 8$ & STLC & $1 \& 4 \& 5$ & STLC \\
\hline $1 \& 4 \& 6$ & STLC & $1 \& 4 \& 7$ & STLC \\
\hline $1 \& 4 \& 8$ & STLC & $1 \& 5 \& 6$ & STLC \\
\hline $1 \& 5 \& 7$ & Inaccessible from zero velocities & $1 \& 5 \& 8$ & STLC \\
\hline $1 \& 6 \& 7$ & STLC & $1 \& 6 \& 8$ & STLC \\
\hline $1 \& 7 \& 8$ & Inaccessible from zero velocities & & \\
\hline
\end{tabular}

This analysis shows that the attitude of the PNPNPNPN star-shaped octorotor is STLC for all two-motors failures. For three and four motors failures, the results are stored in Tables 8 and 9 respectively. The experimental applicability of these results will be discussed later.

Table 8: STLC analysis of the PNPNPNPN star-shaped octorotor after three motors failures. 


\begin{tabular}{|c|c|c|c|}
\hline Motors failures & Controllability & Motors failures & Controllability \\
\hline $1 \& 2 \& 3 \& 4$ & Inaccessible from zero velocities & $1 \& 2 \& 3 \& 5$ & Inaccessible from zero velocities \\
\hline $1 \& 2 \& 3 \& 6$ & Inaccessible from zero velocities & $1 \& 2 \& 3 \& 7$ & Inaccessible from zero velocities \\
\hline $1 \& 2 \& 3 \& 8$ & Inaccessible from zero velocities & $1 \& 2 \& 4 \& 5$ & STLC \\
\hline $1 \& 2 \& 4 \& 6$ & Inaccessible from zero velocities & $1 \& 2 \& 4 \& 7$ & STLC \\
\hline $1 \& 2 \& 4 \& 8$ & Inaccessible from zero velocities & $1 \& 2 \& 5 \& 6$ & STLC \\
\hline $1 \& 2 \& 5 \& 7$ & Inaccessible from zero velocities & $1 \& 2 \& 5 \& 7$ & Inaccessible from zero velocities \\
\hline $1 \& 2 \& 6 \& 7$ & STLC & $1 \& 2 \& 6 \& 8$ & Inaccessible from zero velocities \\
\hline $1 \& 2 \& 7 \& 8$ & Inaccessible from zero velocities & $1 \& 3 \& 4 \& 5$ & Inaccessible from zero velocities \\
\hline $1 \& 3 \& 4 \& 6$ & STLC & $1 \& 3 \& 4 \& 7$ & Inaccessible from zero velocities \\
\hline $1 \& 3 \& 4 \& 8$ & STLC & $1 \& 3 \& 5 \& 6$ & Inaccessible from zero velocities \\
\hline $1 \& 3 \& 5 \& 7$ & Inaccessible from zero velocities & $1 \& 3 \& 5 \& 8$ & Inaccessible from zero velocities \\
\hline $1 \& 3 \& 6 \& 7$ & Inaccessible from zero velocities & $1 \& 3 \& 6 \& 8$ & STLC \\
\hline $1 \& 3 \& 7 \& 8$ & Inaccessible from zero velocities & $1 \& 4 \& 5 \& 6$ & Inaccessible from zero velocities \\
\hline $1 \& 4 \& 5 \& 7$ & Inaccessible from zero velocities & $1 \& 4 \& 5 \& 8$ & STLC \\
\hline $1 \& 4 \& 6 \& 7$ & STLC & $1 \& 4 \& 6 \& 8$ & Inaccessible from zero velocities \\
\hline $1 \& 4 \& 7 \& 8$ & Inaccessible from zero velocities & $1 \& 5 \& 6 \& 7$ & Inaccessible from zero velocities \\
\hline $1 \& 5 \& 6 \& 8$ & STLC & $1 \& 5 \& 7 \& 8$ & Inaccessible from zero velocities \\
\hline $1 \& 6 \& 7 \& 8$ & Inaccessible from zero velocities & & \\
\hline
\end{tabular}

Table 9: STLC analysis of the PNPNPNPN star-shaped octorotor after four motors failures.

\subsection{Discussion}

The analysis presented above considers only the attitude controllability problem. However, the multirotor should also maintain constant altitude after failures occurrence. When trying to guarantee these two conditions, motors limits can be violated. Equilibrium thrusts should be calculated for each multirotor in each failure case in order to study this effect.

Without taking into consideration actuators limits, Table 10 presents a comparison between the different octorotor configurations in terms of controllability after two motors failures.

\begin{tabular}{|c|c|}
\hline Arrangement & \%Controllability \\
\hline Coaxial Counter-rotating & $57.1 \%$ \\
Coaxial Co-rotating & $85.71 \%$ \\
PPNNPPNN star-shaped & $85.71 \%$ \\
PNPNPNPN star-shaped & $100 \%$ \\
\hline
\end{tabular}

Table 10: Controllability comparison for different multirotor arrangements with two rotor failures.

The results show that the PNPNPNPN arrangement is theoretically the best in terms of fault tolerance since the octorotor can maintain stable attitude after all combinations of two motors failures. However, the experimental applicability of these results should also be studied in terms of the used motors and the maximum thrust they can provide. For example, in case of failures of motors 2 and 4 in a PNPNPNPN configuration, the equilibrium states are: $f_{1}=0.58 F, f_{3}=2.82 F, f_{5}=0.58 F, f_{6}=2 F, f_{7}=0, f_{8}=2 F$, 
where $f_{i}$ denotes the commanded thrust of motor $i$, and $F$ is the thrust provided by each motor in nominal flight. It is possible that $f_{3}$ exceeds the thrust limit of the actuator and the equilibrium could not be implemented on the octorotor.

On the other hand, the controllability analysis has shown that the three presented octorotor configurations are better than the coaxial counter-rotating octorotor in terms of fault tolerance capabilities. However when designing a multirotor vehicle, there are other criteria to be taken into consideration. For example, compared to the second configuration, the coaxial octorotor has better thrust since two coaxial rotors spinning in the same direction interfere with each other (Rinaldi (2014)). Compared to the star-shaped configurations, a coaxial octorotor has advantages in terms of the UAV size. A classical star octorotor needs more arms, and each arm needs to be longer to guarantee adequate spacing among the rotors.

\section{Control Mixing and State Feedback Control}

The state feedback control law used in both normal and degraded situations for the attitude stabilization is the PD controller with saturations for the roll and pitch control and the PID controller for the yaw control. Both make use of information obtained from the Inertial Measurement Unit (IMU).

$$
\begin{gathered}
\tau_{\phi}=\frac{I_{x x}}{g}\left[\sigma_{p y}\left(k_{p y}\left(y-y_{d}\right)\right)+\sigma_{d y}\left(k_{d y} \dot{y}\right)-\right. \\
\left.\sigma_{p \phi}\left(k_{p \phi} \phi\right)-\sigma_{d \phi}\left(k_{d \phi} \dot{\phi}\right)\right] \\
\tau_{\theta}=-\frac{I_{y y}}{g}\left[\sigma_{p x}\left(k_{p x}\left(x-x_{d}\right)\right)+\sigma_{d x}\left(k_{d x} \dot{x}\right)-\right. \\
\left.\sigma_{p \theta}\left(k_{p \theta} \theta\right)-\sigma_{d \theta}\left(k_{d \theta} \dot{\theta}\right)\right] \\
\tau_{\psi}=K_{p} e+K_{d} \dot{e}+K_{I} \int_{0}^{t} e(\tau) d \tau
\end{gathered}
$$

All the terms $k_{\alpha \gamma}$ and $K_{\beta}$ are the controller's positive gains. $\sigma_{p y}, \sigma_{d y}, \sigma_{p \phi}$, $\sigma_{d \phi}, \sigma_{p x}, \sigma_{d x}, \sigma_{p \theta}$, and $\sigma_{d \theta}$ are saturation functions defined as follows:

$$
\begin{cases}\sigma_{b_{i}}(s)=b_{i} & \text { if } \quad s>b_{i} \\ \sigma_{b_{i}}(s)=s & \text { if } \quad-b_{i} \leq s \leq b_{i} \\ \sigma_{b_{i}}(s)=-b_{i} & \text { if } \quad s<-b_{i}\end{cases}
$$

After a failure occurrence, the attitude is stabilized via a state feedback control law and a reconfiguration of the control mixing. A set of control mixing laws, each matching a fault situation, are computed offline by resolving a constrained non linear optimization problem, see (Saied et al. (2015)). 


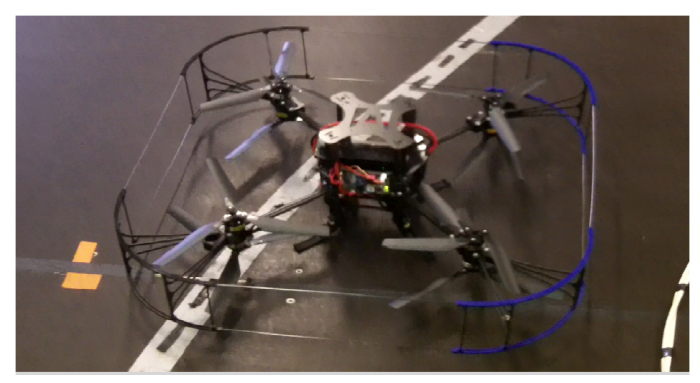

Figure 4: The experimental Octorotor

Based on the output of a Fault Detection and Isolation (FDI) module, the corresponding law is applied.

\section{Experimental Validation}

The control mixing and the feedback control law introduced above were coded in $\mathrm{C}++$ and downloaded on the IGEP microcontroller to be run onboard. Different experimental tests are presented to demonstrate the effectiveness of the designed control system and to validate the controllability analysis.

\subsection{Experimental System}

The Robotex coaxial octorotor UAV of the Heudiasyc laboratory is shown in Figure 4. It uses Bl2827 - 35 brushless motors driven with BLCTRLV2 controllers (Mikrokopter) giving motors speeds measurements. It is equipped with a Microstrain 3DMGX3 - 25 Inertial Measurement Unit (IMU) composed of accelerometer, gyroscope, and magnetometer sensors giving Euler angles and rotation speed measurements at $100 \mathrm{~Hz}$, and an ultrasonic sensor SRF08 giving altitude measurements. The control law is executed in real time onboard the vehicle. The UAV program is connected to a ground station where the parameters (control laws, filters...) are tuned during the system development.

The octorotor inertia was extracted from the software Catia and was found to be as follows: $I_{x x}=I_{y y}=4.2 * 10^{-2} \mathrm{Kg} \cdot \mathrm{m}^{2}, I_{z z}=7.5 * 10^{-2} \mathrm{Kg} \cdot \mathrm{m}^{2}$. The propeller inertia was neglected. The vehicle mass was measured to be $1.6 \mathrm{~kg}$, and the distance from the center of mass to the center of the propellers is $l=0.23 m$. The propellers were characterized using a force/torque sensor. 
The thrust and reaction torque coefficients were estimated as $K_{f}=3 *$ $10^{-5} \mathrm{Ns}^{2} / \mathrm{rad}^{2}$ and $K_{t}=7 * 10^{-7} \mathrm{Nm} / \mathrm{rad}^{2}$.

\subsection{Activity and Fault Injection}

Different experiments were conducted where one, two, three and four motors failures are considered:

1. Failure of motor 6 ;

2. Failures of motors 6 and 2;

3. Failures of motors 6 and 4;

4. Failures of motors 6 and 1 ;

5. Failures of motors 6,2 and 4;

6. Failures of motors 6, 2, 4 and 8;

These scenarios represent all the non symmetric cases where the octorotor was shown to be STLC.

To illustrate a total failure in the propeller system, a motor is turned off by setting its power to zero from the ground station or by remote control.

\subsection{Results}

In these scenarios, the octorotor is brought to a hovering stable flight, then the failures are injected. Due to the lack in space, only the results of the experiment 6 will be presented where four failures are injected successively. The other experiments are shown in the video accompanying the paper [https : //youtu.be/P6o_RFQGpps].

The motors $6,2,4$ and 8 are turned off successively from the ground station, at times $t_{6}=33.37 \mathrm{~s}, t_{2}=41.05 \mathrm{~s}, t_{4}=49.2 \mathrm{~s}$, and $t_{8}=57.6 \mathrm{~s}$. An FDI module was also implemented, so that the control mixing was reconfigured after $0.23 \mathrm{~s}$ and $0.84 \mathrm{~s}$ for motors 6 and 2 respectively and automatically after 1 s without diagnosis for motors 4 and 8 . The position in $x$ and $y$ of the octorotor is controlled using a motion capture system.

The motors speeds are shown in Fig. 2. The altitude and angular speeds are presented in Fig. 3 and 4 . The results validate the controllability results deduced above from the analysis, and the possibility to stabilize the octorotor by a continuous state feedback law after one or more motors failures. This is a motivation for using multirotors with redundant actuators instead of using quadrotors. 


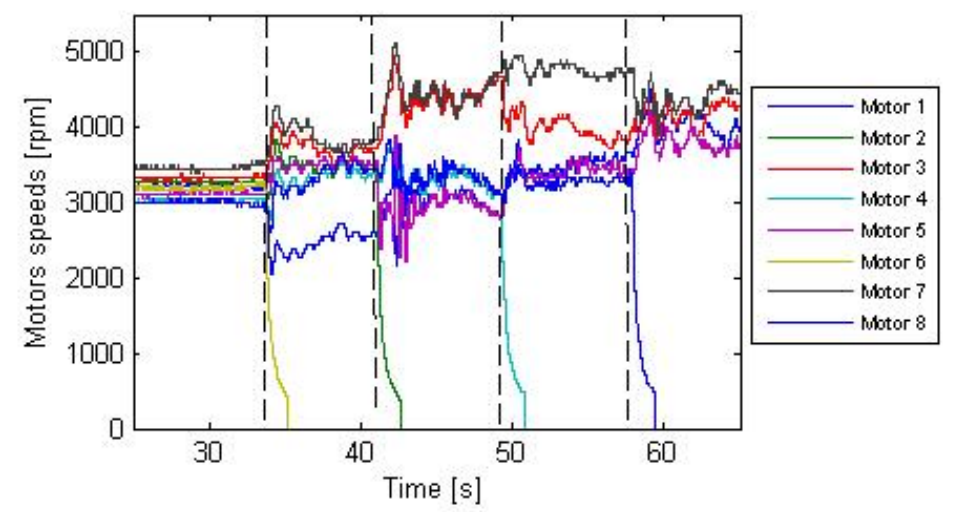

Figure 5: Motors speeds after four motors failures [rpm]; Faults are injected respectively on motors $6,2,4$ and 8 at times $t_{6}=33.73 \mathrm{~s}, t_{2}=41.05 \mathrm{~s}, t_{4}=49.2 \mathrm{~s}$ and $t_{8}=57.6 \mathrm{~s}$.

The dashed lines indicate the fault injections times

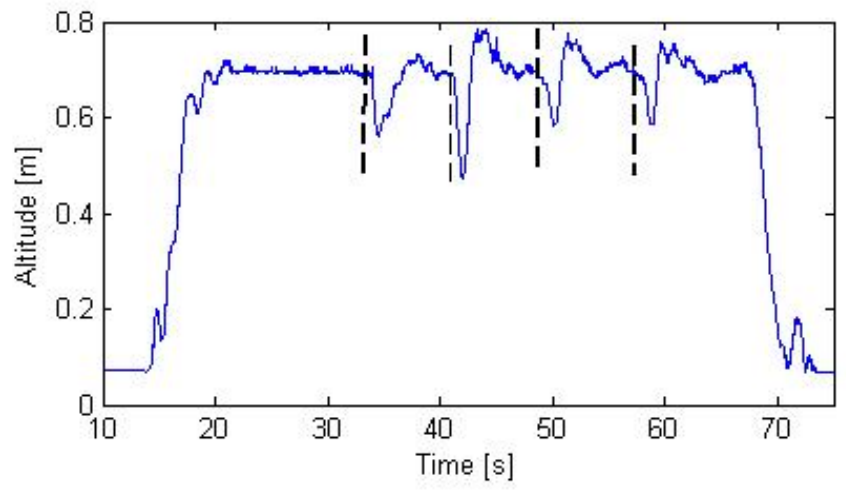

Figure 6: Altitude after four motors failure [m]; The octorotor takes off at time $\mathrm{t}=15 \mathrm{~s}$, then faults are injected respectively on motors $6,2,4$ and 8 at times $t_{6}=33.73 \mathrm{~s}, t_{2}=$ $41.05 \mathrm{~s}, t_{4}=49.2 \mathrm{~s}$ and $t_{8}=57.6 \mathrm{~s}$. The octorotor lands at time $\mathrm{t}=68 \mathrm{~s}$. The dashed lines indicate the fault injections times 

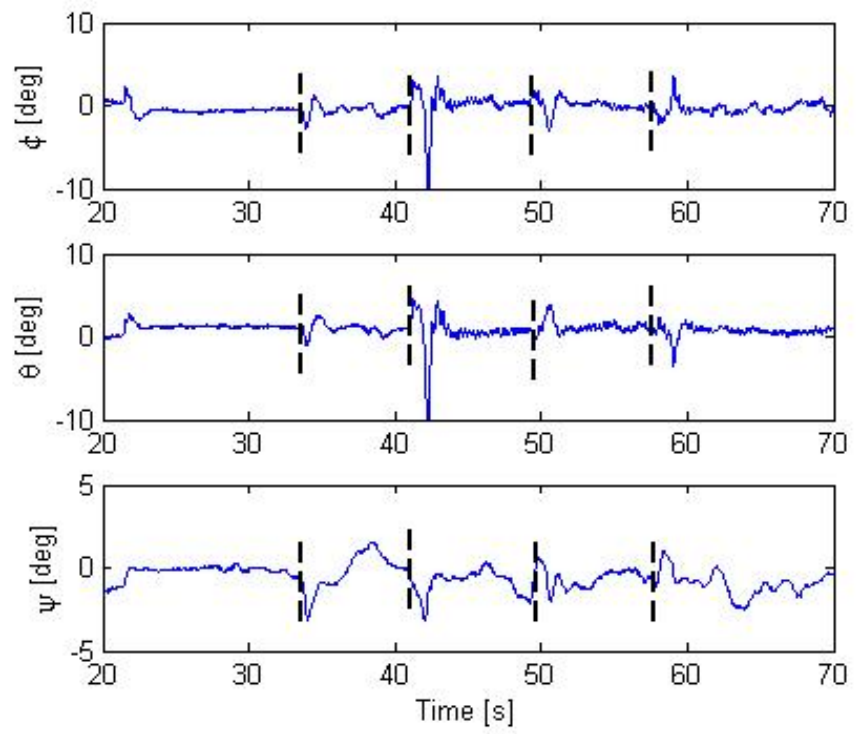

Figure 7: Euler angles after four motors failure [deg]. Faults are injected respectively on motors $6,2,4$ and 8 at times $t_{6}=33.73 \mathrm{~s}, t_{2}=41.05 \mathrm{~s}, t_{4}=49.2 \mathrm{~s}$ and $t_{8}=57.6 \mathrm{~s}$. The dashed lines indicate the fault injections times. 


\section{Conclusions}

A method has been applied to assess the controllability of multirotor systems under actuators failures, based on the small time local controllability theory with unilateral control inputs. This study is useful to establish the existence of continuous control laws that can asymptotically stabilize the system around the equilibrium. Analysis results have shown that octorotors with different rotor configurations (coaxial or star-shaped) have different fault tolerant capabilities. For example, a coaxial counter-rotating octorotor can maintain roll, pitch and yaw control for $55.5 \%$ of all fault configurations up to two random rotor failures and for $23.4 \%$ of fault configurations up to any four rotor failures. The control of the reduced attitude (without yaw control, see section 4.1.1) is possible for all the 162 possible fault combinations considering that at least four motors are healthy.

An actuator failure recovery technique has been applied to this coaxial octorotor for scenarios representing all the non symmetric cases where the octorotor was shown to be STLC. Although the design and the study in this paper are specifically aimed at the octorotor application, they can be applied to any multirotor with actuator redundancy.

The controllability study presented in this paper was applied to the nonlinear model of the octorotor attitude, however, a comparative study shows that the results obtained in this paper, are equivalent to those obtained from the application of the linear theories (Heemels and Camlibel (2007)), on the linearized model around the equilibrium, that consider the constraints on the inputs. This can be explained by the fact that, in this paper, the controllability was studied near zero velocity states, and that the control vectors $g_{i}$ are constant and independent of the variable states. In future works, we intend to apply this theory for the controllability of the octorotor under motors failures when following trajectories.

\section{ACKNOWLEDGEMENTS}

This work was carried out and funded in the framework of the Labex MS2T (Reference ANR-11-IDEX-0004-02) and the ROBOTEX Equipment of Excellence (Reference ANR-10- EQPX-44). They were supported by the French Government, through the program Investments for the future managed by the National Agency for Research.

This work has been partially funded with support from the National Council for Scientific Research in Lebanon (CNRSL). 
The authors also express their gratitude to Guillaume Sanahuja and Gildas Bayard, engineers at Heudiasyc Laboratory, for their support in performing our real-time experiments.

\section{References}

Y. Zhang, A. Chamseddine, C. Rabbath, B. Gordon, C.-Y. Su, S. Rakheja, C. Fulford, J. Apkarian, and P. Gosselin, "Development of advanced FDD and FTC techniques with application to an unmanned quadrotor helicopter testbed," Journal of the Franklin Institute, vol. 350, no. 9, pp. 2396-2422, 2013.

A. Chamseddine, Y. Zhang, C. A. Rabbath, C. Join, and D. Theilliol, "Flatness-based trajectory planning/replanning for a quadrotor unmanned aerial vehicle," IEEE Transactions on Aerospace and Electronic Systems, vol. 48, no. 4, pp. 2832-2848, 2012.

M. Ranjbaran and K. Khorasani, "Fault recovery of an under-actuated quadrotor aerial vehicles," IEEE Conference on Decision and Control (CDC), Atlanta, Dec. 15-17, 2010, pp. 4385-4392.

M.W. Mueller and R. D Andrea, "Stability and control of a quadrocopter despite the complete loss of one, two, or three propellers," IEEE International Conference on Robotics and Automation (ICRA), Hong Kong, China, May 31-June 7, 2014, pp. 45-52.

M. Saied, B. Lussier, I. Fantoni, C. Francis, H. Shraim and G. Sanahuja, "Fault Diagnosis and Fault-Tolerant Control Strategy for Rotor Failure in an Octorotor," IEEE International Conference on Robotics and Automation (ICRA), Washington, DC, USA, May 26-30, 2015.

H. Alwi and C. Edwards, "Fault Tolerant Control of an Octorotor Using LPV based Sliding Mode Control Allocation," American Control Conference (ACC), Washington, DC, USA, June 17-19, 2013, pp. 6505- 6510.

J. M. Coron, "Control and Nonlinearity," Mathematical Surveys and Monographs, American Mathematical Society, 2007.

E. D. Sontag, "Kalmans Controllability Rank Condition: From Linear to Nonlinear," Mathematical System Theory, chapter 7, pp. 453-462, 1991. 
H. J. Sussmann, "A general theorem on local controllability," SIAM Journal on Control and Optimization, vol. 25, no. 1, pp. 158-194, 1987.

B. Goodwine, J. Burdick, "Controllability with Unilateral Control Inputs", IEEE International Conference on Decision and Control (CDC), Kobe, Dec. 11-13, 1996, pp. 3394-3399.

T. Schneider, G. Ducard, K. Rudin and P. Strupler, "Fault-tolerant Control Allocation for Multirotor Helicopters Using Parametric Programming," International Micro Air Vehicle Conference and Flight Competition, Braunschweig, Germany, July, 2012.

G. Du, Q. Quan and K. Cai, "Controllability Analysis and Degraded Control for a Class of Hexacopters Subject to Rotor Failures", Journal of Intelligent Robotic Systems, vol. 78, issue 1, pp. 143-157, September 2014.

R. F. Brammer, "Controllability in Linear Autonomous Systems With Positive Controllers," SIAM Journal on Control, vol. 10, No. 2, pp. 339-353, 1972.

M. Saied, B. Lussier, I. Fantoni, C. Francis and H. Shraim, "Fault Tolerant Control for Multiple Successive Failures in an Octorotor: Architecture and Experiments," IEEE International Conference on Intelligent Robots and Systems, Hamburg, Germany, September 2015.

F. Rinaldi, "Automatic control of a multirotor," PhD Thesis, Politecnico di Torino, April 2014.

W.P.M.H Heemels and M.K. Camlibel, "Controllability of Linear Systems with Input and State Constraints", IEEE International Conference on Decision and Control (CDC), New Orleans, LA, USA, Dec. 12-14, 2007. 